\title{
First Note on the Definition of $s_{1}$-Convexity
}

\author{
I. M. R. Pinheiro \\ P.O. Box 12396 A'Beckett St, Melbourne, Victoria, Australia, 8006 \\ Email: illmrpinheiro@gmail.com
}

Received 25 November 2014; revised 18 December 2014; accepted 22 December 2014

Copyright (C) 2014 by author and Scientific Research Publishing Inc.

This work is licensed under the Creative Commons Attribution International License (CC BY).

http://creativecommons.org/licenses/by/4.0/

(c) (i) Open Access

\section{Abstract}

In this note, we analyze a few major claims about $K_{s}^{1}$. As a consequence, we rewrite a major theorem, nullify its proof and one remark of importance, and offer a valid proof for it. The most important gift of this paper is probably the reasoning involved in all: We observe that a constant, namely $t$, has been changed into a variable, and we then tell why such a move could not have been made, we observe the discrepancy between the claimed domain and the actual domain of a supposed function that is created and we then explain why such a function could not, or should not, have been created, along with others.

\section{Keywords}

Analysis, Convexity, Definition, $S$-Convexity, Geometry

\section{Introduction}

$K_{s}^{1}$ is a very interesting component of $S$-convexity, not to say exotic: It differs substantially from $K_{s}^{2}$, yet, in a certain sense, seems to supplement it.

\subsection{Notation}

We use the symbols from [1] here:

- $K_{s}^{1}$ for the class $s$-convex functions in the first sense, where $s \in \mathfrak{R} / 0<s \leq 1$;

- $K_{s}^{2}$ for the class $s$-convex functions in the second sense, where $s \in \mathfrak{R} / 0<s \leq 1$;

- $K_{0}$ for the class convex functions;

- $s_{1}$ for the variable $s, 0<s_{1} \leq 1$, used for the first type of $s$-convexity;

- $s_{2}$ for the variable $s, 0<s_{2} \leq 1$, used for the second type of $s$-convexity.

Remark 1 The class 1-convex functions is simply a subclass of the class convex functions. If we make the domain of the convex functions be inside of the set of the non-negative real numbers, we then have the class 
1-convex functions: $K_{1}^{1} \equiv K_{1}^{2} \equiv K_{0}$.

\subsection{Definition}

We use the definition from [1] here:

Definition 1 A function $f: X \rightarrow \Re$ is said to be $s_{1}$-convex if the inequality

$$
f\left(\lambda^{\frac{1}{s}} x+(1-\lambda)^{\frac{1}{s}} y\right) \leq \lambda f(x)+(1-\lambda) f(y)
$$

holds $\forall \lambda / \lambda \in[0,1] ; \forall x, y \in X ; \quad X \subset \mathfrak{R}_{+}$.

Remark 2 If the inequality is obeyed in the reverse ${ }^{1}$ situation by $f$, then $f$ is told to be $s_{1}$-concave.

\subsection{Theorems That We Discuss Here}

Dragomir and Pearce, in [2], state that Hudzik and Maligranda, in [3], told us that:

Theorem 1.1 Let $0<s<1$. If $f \in K_{s}^{1}$, then $f$ is nondecreasing on $(0, \infty)$ and $\lim _{u \rightarrow 0^{+}} f(u) \leq f(0)$. We can infer, from the above theorem, that:

(1) (Claim X) Any function in $K_{s}^{1}$, with domain contained in $(0, \infty), s$ specified, $s \neq 1$, is nondecreasing;

(2) (Claim Y) $\lim _{u \rightarrow 0^{+}} f(u) \leq f(0)$ for $f \in K_{s}^{1}, \quad s \neq 1$.

In this paper, we prove that (1) is true but does not yet have an actual proof and (2) is incomplete, controversial or unnecessary.

\section{Analyzing Claim X}

[2] presents the following sequence of implications as a proof for the claim X:

Proof. We have, for $u>0$ and $\alpha \in[0,1]$,

(PROBLEM 1) $f\left[\left(\alpha^{\frac{1}{s}}+(1-\alpha)^{\frac{1}{s}}\right) u\right] \leq \alpha f(u)+(1-\alpha) f(u)=f(u)$.

The function

$$
h(\alpha)=\alpha^{\frac{1}{s}}+(1-\alpha)^{\frac{1}{s}}
$$

is continuous on $[0,1]$, decreasing on $\left[0, \frac{1}{2}\right]$, increasing on $\left[\frac{1}{2}, 1\right]$ and

(PROBLEM 2) $h([0,1])=\left[h\left(\frac{1}{2}\right), h(1)\right]=\left[2^{1-\frac{1}{s}}, 1\right]$.

This yields that

(5.147) $f(t u) \leq f(u)$ for all $u>0, t \in\left[2^{1-\frac{1}{s}}, 1\right]$.

If now $t \in\left[2^{1-\frac{1}{s}}, 1\right]$, then $t^{\frac{1}{2}} \in\left[2^{1-\frac{1}{s}}, 1\right]$, and therefore, by the fact that (5.147) holds for all $u>0$, we get $f(t u)=f\left(t^{\frac{1}{2}}\left(t^{\frac{1}{2}} u\right)\right) \leq f\left(t^{\frac{1}{2}}\right) \leq f(u)$ for ${ }^{2}$ all $u>0$. By induction, we therefore obtain that

(5.148) (PROBLEM 3) $f(t u) \leq f(u)$ for all $u>0, t \in(0,1]$.

Hence, by taking $0<u \leq v$ and applying (5.148), we get

${ }^{1}$ Reverse here means $>$, not $\geq$.

${ }^{2}$ Notice that the second member of the inequality should have been $f\left(t^{\frac{1}{2}} u\right)$, not $f\left(t^{\frac{1}{2}}\right)$ as well. 
$f(u)=f\left(\frac{u}{v} \cdot v\right) \leq f(v)$ which means that $f$ is non-decreasing on $(0, \infty)$.

We prove that PROBLEM 1, PROBLEM 2, and PROBLEM 3 will make the proof not be a mathematical proof. There are more problems with the proof, however.

From [4], we learn that we cannot have $x=y$ in the definition of $S$-convexity (it is all tied to the geometric definition of convexity).

This way, PROBLEM 1 should, per se, nullify the proof that we have just presented.

Notwithstanding, notice that $2^{1-\frac{1}{s}}$ goes as close as we wish (PROBLEM 2) to 0 (limit when $s \rightarrow 0$ ) and actually assumes the value 1 (when $s=1$ ), what then makes the interval $\left[2^{1-\frac{1}{s}}, 1\right]$ be a degenerated interval, or not be an interval, but just a point instead. Besides, we have different intervals, depending on the value of $s$ we choose $^{3}(s=0.5 \Rightarrow t \in[0.5,1], \quad s=0.7 \Rightarrow t \in[0.743,1])$. We then know that we cannot generalize this to $t \in(0,1]$. That is a very serious mistake. Besides, $t$ replaces $\alpha^{\frac{1}{s}}+(1-\alpha)^{\frac{1}{s}}$ and is what they themselves have called $h(\alpha)$, therefore a function, what then would have to mean that $t$ is not a constant. That means that we can replace $t$ with numerical values, but those would have to be each and every value of the function $\alpha^{\frac{1}{s}}+(1-\alpha)^{\frac{1}{s}}$ for us to claim that we have $h(\alpha)$.

We do notice that $s<1$ instead of the usual, $s \leq 1$, in the theorem, so that $\left[2^{1-\frac{1}{s}}, 1\right]$ does not run the risk of being a degenerated interval or an improper interval.

Even if the result were true, and we are obviously entitled to try to get it using the reasoning contained in the above proof, we cannot use the just-exposed lines as a proof.

As for PROBLEM 3: Notice that, when $s=0.5, t \in[0.5,1]$. This means that $t \in[0.5,1]$ instead of what is written there at least when $s=0.5$.

With that, we must have $0<v \leq 2 u \leq 2 v$, for instance, in the next line, not $0<u \leq v$, when $s=0.5$. Notice that this sort of problem will happen with all values of $s$.

In this situation, just like in the original situation, in the proof, $v$ and $u$ cannot be variables for $f$ because whatever be a variable for $f$ spans $(0, \infty)$ or its domain set with no discrimination. We select only the values that obey the rule that we have created, which is, after due fixing in what regards $s=0.5,0<v \leq 2 u \leq 2 v$. This way, we are using only a few selected values of the original variable of $f$. Because of that, we should create a new variable, which we could call $w$, which is then going to be equal to $\frac{u}{v}$, with $u$ and $v$ assuming only the values that we have selected, what means that the simplification is not valid in the proof. Consequently, the inference is not valid.

On the other hand, notice that $w=\frac{u}{v}$ is a function that is totally different from

$$
h(\alpha)=\alpha^{\frac{1}{s}}+(1-\alpha)^{\frac{1}{s}}
$$

in shape, so that even if the first coordinates of the functions are the same, say $\frac{u}{v}$ varies from 0 to 1 , the second coordinates may be completely different (take $\frac{u}{v}=0$ and $\alpha=0$, for instance).

We must respect the original function, the one from the definition, when proving something in Mathematics or replace it with a completely equivalent function, what then means that this step is unacceptable (replacing $h(\alpha)$ with $\frac{u}{v}$ and then applying the rule that should apply when we have $f(h(\alpha) u)$ for when we have ${ }^{3} 0.743$ is an approximation. The original value was something like 0.742997 . 
$f\left(\frac{u}{v} v\right)$ instead).

We have then just proven that there is no actual proof of $f$ being nondecreasing on $(0, \infty)$ so far.

It is then the case that we either have to find a proper proof for the claim or a suitable counter-example/proof of the contrary.

Notice that all convex functions whose domain is in $\mathfrak{R}_{+}$should be $s_{1}$-convex ( $K_{s}^{1}$ is supposed to extend $K_{1}^{1}$, so that this should be valid for any $s_{1}$ we choose, provided that $0<s_{1} \leq 1$ ). When $s=1$, we should have precisely the class convex functions.

The quadratic function $f(x)=x^{2}-8 x+16$, in the piece of domain $(0,4]$, is a convex, and therefore should also be an $s_{1}$-convex, function, and it decreases in $(0,4]$. Notwithstanding, $s=1$ is part of the exclusions in this theorem, so that this is not a counter-example to their claim.

A suitable proof would be similar to what has been presented in [2], but not equal.

Notice that $x$ and $y$ can always be made as similar as we wish.

We can make one differ from the other by the thousandth decimal place, for instance.

We can therefore, in practice, equate $x$ to $y$ whilst applying the definition of $s_{1}$-convex function.

Proof. When we apply the definition of $s_{1}$-convexity to a function that satisfy the conditions of this theorem, $\alpha=\frac{1}{2}$ will always be inside of the inclusions, so that we can use it in our proof with no loss.

In replacing $\alpha$ with $\frac{1}{2}$ in our definition, we get $f\left(\frac{x+y}{2^{\frac{1}{s}}}\right) \leq \frac{f(x)+f(y)}{2}$.

In making $y$ go really close to $x$, what we can always do because of $(*)$, which is a condition that we will explain later on in this paper, we can make them differ by the thousandth decimal place, for instance. In this case, in practice, we can equate both.

When we do that, our inequality becomes $f\left(\frac{2 x}{2^{\frac{1}{s}}}\right) \leq \frac{2 f(x)}{2}$ or $f\left(\frac{x}{2^{\frac{1}{s}-1}}\right) \leq f(x)$.

Because $s \neq 1$ in our theorem, we have $0<s<1$ and therefore $\frac{1}{s}>1$, what implies $\frac{1}{s}-1>0$. Assuming $x$ is a nonnegative number (definition of $K_{s}^{1}$ ), we get $\frac{x}{2^{\frac{1}{s}-1}} \leq x$.

In this case, we can only have a nondecreasing function $\left(\frac{x}{2^{\frac{1}{s}-1}} \leq x\right.$ and $\left.f\left(\frac{x}{2^{\frac{1}{s}-1}}\right) \leq f(x)\right)$.

\section{Analyzing Claim Y}

$f(0)$ must exist because it appears in the theorem. Therefore, 0 is part of the domain of the function and we can replace the domain interval $(0, \infty)$ with $[0, \infty)$ at least when stating the second part of the theorem.

Because every $s$ - convex function is continuous (please refer to $(*)$ on page 5 ), we know that

$\lim _{u \rightarrow c^{+}} f(u)=\lim _{u \rightarrow c_{-}} f(u)=f(c)$ for each $c$ in the domain of $f$, therefore

$\lim _{u \rightarrow 0^{+}}^{u \rightarrow c^{+}} f(u)=\lim _{u \rightarrow 0_{-}} f(u)=f(0)$ when both lateral limits can be calculated. Because the domain interval does not include the left neighbors of zero, we can only have the right lateral limit here.

As a consequence, $\lim _{u \rightarrow 0^{+}} f(u) \leq f(0)$ and $\lim _{u \rightarrow 0^{+}} f(u) \geq f(0)$ are both true.

Since it is never true that $\lim _{u \rightarrow 0^{+}} f(u)<f(0)$ or $\lim _{u \rightarrow 0^{+}} f(u)>f(0)$, we should at most write that $\lim _{u \rightarrow 0^{+}} f(u)=f(0)$.

The definition of $K_{s}^{1}$ implies any $f$ that be continuous (simply imagine that it be not. Imagine a discontinuity of first type, right on the vertex of a parabola that has a point of minimum value, which we know is the image of a convex, therefore $S$-convex in both senses (as for all we have been told by others), function. Now let 
$\left(x_{2}, f\left(x_{2}\right)\right)$, where $x_{2}$ is the first coordinate of the vertex of the parabola we talk about, be a point that is sufficiently distant from the vertex and assume that $x_{2} \in D_{f}$. In fact, make this point be fully detached from the rest of the graph and lie miles below it. Make it be the only atypical point in this parabola. Now make $f\left(x_{1}\right)$, $x_{1} \leq x_{2}, \quad x_{1} \in D_{f}$, be our $f(x)$ in the definition inequality for $K_{s}^{1}$ and $f\left(x_{2}\right)$ be our $f(y)$. Notice that we will unavoidably find points in the graph that are above the limiting line, and that will make the function be both not convex and not $S$-convex, what is absurd). Please call this paragraph $(*)$.

We will also try to find the mistake in the proof presented in [2].

The proof in [2] is:

Proof. For $u>0$, we have

(PROBLEM 4) $f(\alpha u)=f(\alpha u+\beta 0) \leq \alpha^{s} f(u)+\beta^{s} f(0)$

and making $u \rightarrow 0^{+}$, we obtain

(PROBLEM 5) $\lim _{u \rightarrow 0^{+}} f(u) \leq \lim _{u \rightarrow 0^{+}} f(\alpha u) \leq \alpha^{s} \lim _{u \rightarrow 0^{+}} f(u)+\beta^{s} f(0)$.

Hence,

$$
\lim _{u \rightarrow 0^{+}} f(u) \leq f(0) .
$$

PROBLEM 4 is that the assertion is only true if $\alpha$ and $\beta$ satisfy the conditions of the definition of $K_{s}^{1}$, that is, if $\alpha^{s}+\beta^{s}=1$, what, as we know, implies $0 \leq \alpha \leq 1$ and $0 \leq \beta \leq 1$ [5].

Even if such a piece of information were not relevant to the proof, we can only accept the proof as a proof if such constraints are mentioned.

PROBLEM 5 is that it is missing explaining where the information $f(u) \leq f(\alpha u)$ came from, for instance. Since $\alpha u \leq u$ and their assumption was that the function, in this situation, does not decrease (see (5.147)), what implies that $f(\alpha u) \leq f(u)$, not the opposite, things are unacceptable from this point onwards in the proof.

\section{Supplementary Remarks}

Still in [2], we find a remark that is told to be in [3]:

Remark 3 If $0<s<1$, then the function $f \in K_{s}^{1}$ is nondecreasing on $(0, \infty)$ but not necessarily on $[0, \infty)$.

From Real Analysis, we know that this remark is absurd. It is not possible that one point, in a continuous function, change the nature of the function from nondecreasing to decreasing. That can only happen to a function with a discontinuity on $x=0$.

Suppose that $f: X \subseteq \mathfrak{R}_{+} \rightarrow \mathfrak{R}$ is continuous and nondecreasing on $(0, \infty)$ but not on $[0, \infty)$.

Then, for $0<x_{1}<x_{2}<x_{3}$, we have $f\left(x_{1}\right) \leq f\left(x_{2}\right) \leq f\left(x_{3}\right)$ and $f(0)>f\left(x_{1}\right) \quad\left(\sim\left(f(0) \leq f\left(x_{1}\right)\right)\right)$.

We can then find $x_{1}, x_{1}$ extremely close to zero but different from it, such that $f\left(x_{1}\right)<f(0)$ but $x_{1}>0$.

That would mean that $\exists \epsilon>0$ such that $x_{1} \in(0-\epsilon, 0+\epsilon)$ and $f\left(x_{1}\right)=f(0)-\Delta, \Delta>0$. By assumption of the proposal, however, such a family, of $f\left(x_{1}\right)$ s, could not exist, since its existence would imply that $f \in K_{s}^{1}$ is nondecreasing on $(\epsilon, \infty)$, where $\epsilon$ is greater than zero, instead of on $(0, \infty)$.

This conclusion just makes sense, since saying that $K_{s}^{1}$ functions do not have to be continuous would make sustaining that they extend the class convex functions be a likely-to-be-impossible task (see $(*)$ on page 5 ).

Besides, the definition of $K_{s}^{1}$ does not allow us to do that.

\section{Conclusions}

In this paper, we have rewritten the proof of the theorem

Theorem 5.1 Let $0<s<1$. If $f \in K_{s}^{1}$, then $f$ is nondecreasing on $(0, \infty)$ and $\lim _{u \rightarrow 0^{+}} f(u) \leq f(0)$ and the own theorem as well.

This theorem appears in [2] and is there reported to have originated in [3]. We have proved that if $\lim _{u \rightarrow 0^{+}} f(u) \leq f(0)$, then $\lim _{u \rightarrow 0^{+}} f(u) \geq f(0)$ as well since $\lim _{u \rightarrow 0^{+}} f(u)=f(0)$ for any s-convex function that be defined on $(0, \infty)$ or in one of its subsets and also on zero.

It does not make sense making a theorem to tell the just-written information because we aim objectivity and clarity in Mathematics and the only actual piece of information that we need to know regarding this is contained 
in the definition in an almost explicit way (continuity).

The new version of the theorem is therefore

Theorem 5.2 Let $0<s<1$. If $f \in K_{s}^{1}$, then $f$ is nondecreasing on $(0, \infty)$.

Its new proof is

Proof. When we apply the definition of $s_{1}$-convexity to a function that satisfy the conditions of this theorem, $\alpha=\frac{1}{2}$ will always be inside of the inclusions, so that we can use it in our proof with no loss.

In replacing $\alpha$ with $\frac{1}{2}$ in our definition, we get $f\left(\frac{x+y}{2^{\frac{1}{s}}}\right) \leq \frac{f(x)+f(y)}{2}$.

In making $y$ go really close to $x$, what we can always do because of $(*)$, we can make them differ by the thousandth decimal place, for instance. In this case, in practice, we can equate both.

When we do that, our inequality becomes $f\left(\frac{2 x}{2^{\frac{1}{s}}}\right) \leq \frac{2 f(x)}{2}$ or $f\left(\frac{x}{2^{\frac{1}{s}-1}}\right) \leq f(x)$.

Because $s \neq 1$ in our theorem, we have $0<s<1$ and therefore $\frac{1}{s}>1$, what implies $\frac{1}{s}-1>0$. Assuming $x$ is a nonnegative number (definition of $K_{s}^{1}$ ), we get $\frac{x}{2^{\frac{1}{s}}} \leq x$.

In this case, we can only have a nondecreasing function $\left(\frac{x}{2^{\frac{1}{s}}} \leq x\right.$ and $\left.f\left(\frac{x}{2^{\frac{1}{s}-1}}\right) \leq f(x)\right)$.

We have also nullified a remark of importance ([2]):

Remark 4 If $0<s<1$, then the function $f \in K_{s}^{1}$ is nondecreasing on $(0, \infty)$ but not necessarily on $[0, \infty)$.

The remark has been nullified in this paper in what regards continuous functions and, therefore, unless we change the definitions of convex and $s$-convex functions in order not to have only continuous functions inside of our sets, when one could then think of reassessing this remark, this remark has been nullified in full.

\section{References}

[1] Pinheiro, M.R. (2008) Convexity Secrets. Trafford, Canada, ISBN 1-4251-3821-7.

[2] Pearce, C.E.M. and Dragomir, S.S. (2000) Selected Topics on Hermite-Hadamard Inequalities and Applications. RGMIA Monographs. http://rgmia.org/papers/monographs/Master.pdf

[3] Hudzik, H. and Maligranda, L. (1994) Some Remarks on s-Convex Functions. Aequationes Mathematicae, 48, 100111. http://dx.doi.org/10.1007/BF01837981

[4] Pinheiro, M.R. (2013) Minima Domain Intervals and the S-Convexity, as Well as the Convexity, Phenomenon. Advances in Pure Mathematics, 3, 457-458.

[5] Pinheiro, M.R. (2004) Exploring the Concept of s-Convexity. Proceedings of the 6th WSEAS Int. Conf. on Mathematics and Computers in Physics (MCP '04). 
Scientific Research Publishing (SCIRP) is one of the largest Open Access journal publishers. It is currently publishing more than 200 open access, online, peer-reviewed journals covering a wide range of academic disciplines. SCIRP serves the worldwide academic communities and contributes to the progress and application of science with its publication.

Other selected journals from SCIRP are listed as below. Submit your manuscript to us via either submit@scirp.org or Online Submission Portal.
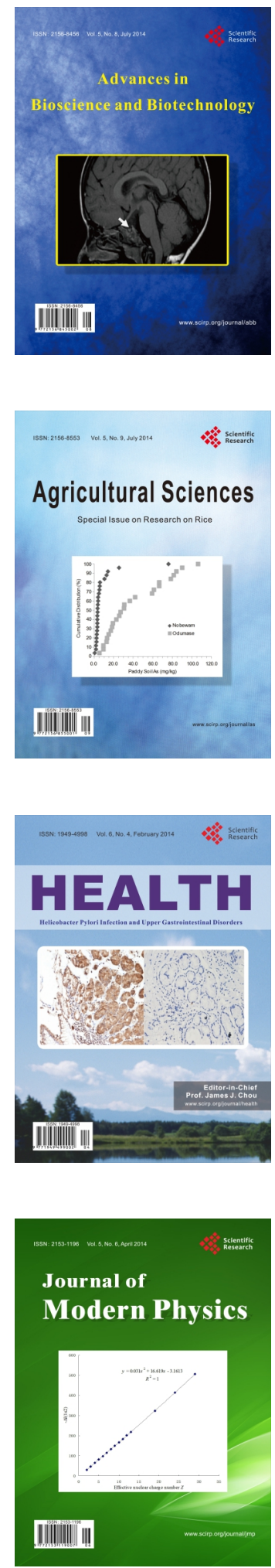
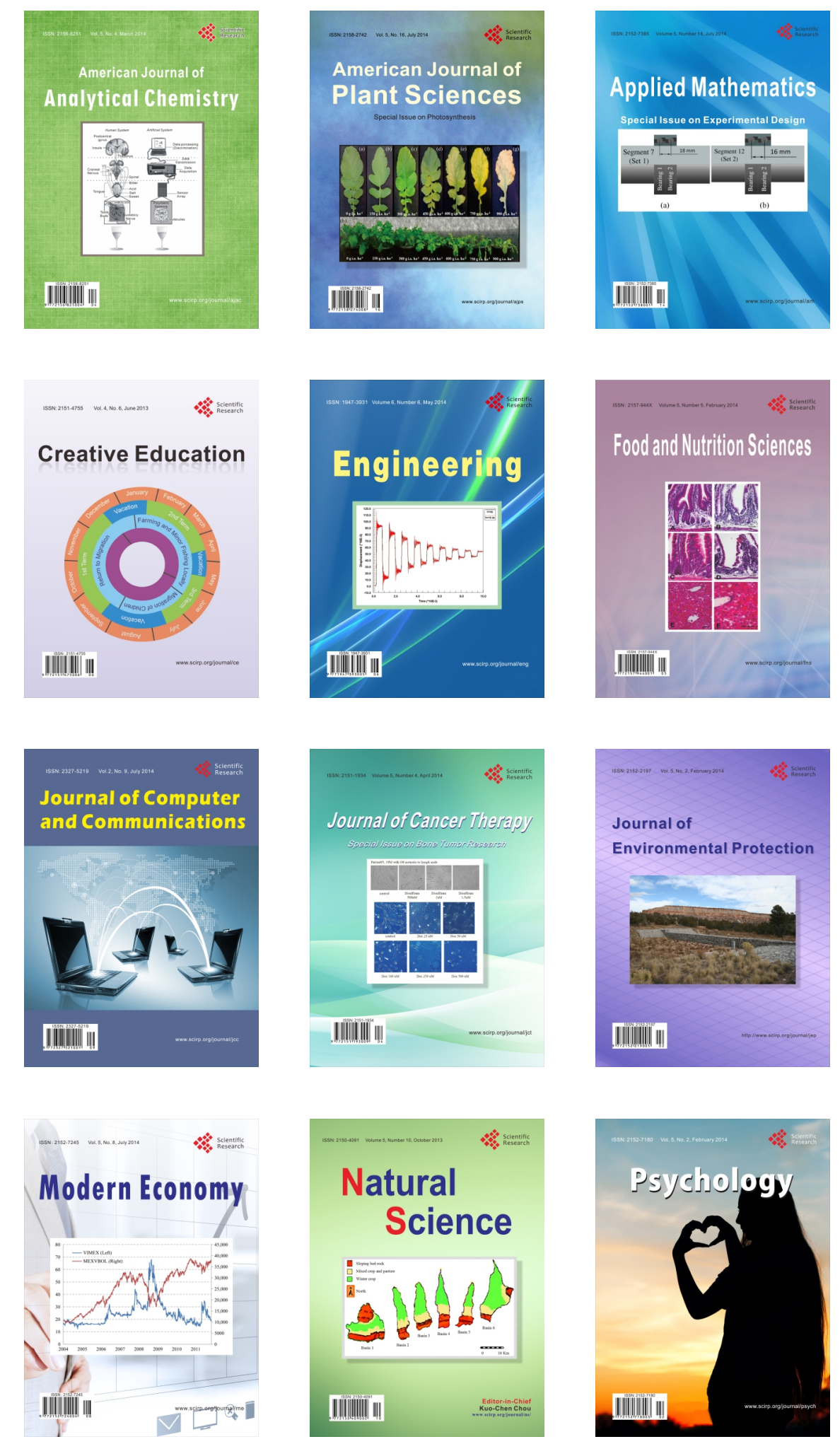